\title{
Composition and strain dependence of the piezoelectric coefficients in $\operatorname{In}_{x} \mathrm{Ga}_{1-x} \mathrm{As}$ alloys
}

\author{
M. A. Migliorato,* D. Powell, and A. G. Cullis \\ Department of Electronic and Electrical Engineering, University of Sheffield, Mappin Street, Sheffield S1 3JD, United Kingdom \\ T. Hammerschmidt \\ Fritz-Haber-Institut der Max-Planck-Gesellschaft, Faradayweg 4-6, 14195 Berlin, Germany \\ G. P. Srivastava \\ School of Physics, University of Exeter, Stocker Road, Exeter EX4 4QL, United Kingdom
}

(Received 2 October 2006; revised manuscript received 13 October 2006; published 29 December 2006)

\begin{abstract}
We address the issue of the composition and strain dependence of the piezoelectric effect in semiconductor materials, which is manifested by the appearance of an electric field in response to shear crystal deformation. We propose a model based on expressing the direct and dipole contributions to the polarization in terms of microscopic quantities that can be calculated by density functional theory. We show that when applied to the study of $\operatorname{In}_{x} \mathrm{Ga}_{1-x}$ As alloys, the model is able to explain and accurately predict the often-observed discrepancies between the experimentally deduced values of $e_{14}$ and those linearly interpolated between the values of InAs and GaAs. The values of the piezoelectric coefficient predicted by our approach compare very well with values deduced from photocurrent measurements of quantum well samples grown on (111) GaAs substrates by molecular beam epitaxy.
\end{abstract}

DOI: 10.1103/PhysRevB.74.245332

PACS number(s): 77.65.Bn, 71.15.-m, 77.65.Ly, 78.67.De

\section{INTRODUCTION}

The piezoelectric effect ${ }^{1,2}$ in bulk semiconductor crystals is a well understood phenomenon, and it finds widespread usage in devices such as ultrasonic transducers, actuators, cantilever systems, and micropositioners. In epitaxially grown semiconductors, the piezoelectric effect can be observed via the off-diagonal strain tensors that exist in quantum wells grown on (111) substrates, quantum wires, and quantum dots. Piezoelectric effects in such nanostructures have attracted a substantial amount of interest in recent years ${ }^{3-5}$ and were identified as the source of experimentally observable optical anisotropies. ${ }^{6-8}$ Previous work has considered only the first-order piezoelectric effects and employed either the experimental piezoelectric coefficients of bulk InAs and bulk GaAs or linear interpolations between the two compounds. A recent study by Bester et al..$^{9}$ has pointed out that neglecting the second-order effects leads to substantial errors in the calculations of quantities such as the piezoelectric field. The authors used self-consistent density functional theory (DFT) and a linear response technique to obtain estimates of the first- and second-order piezoelectric tensor. Based on these results, the authors manifested doubts in the bulk experimental values of the piezoelectric coefficient reported in the literature $\left(-0.045\right.$ and $-0.16 \mathrm{C} / \mathrm{m}^{2}$ for InAs and GaAs, respectively ${ }^{10}$ ), which are predicted according to the model being -0.115 and $-0.230 \mathrm{C} / \mathrm{m}^{2}$. In this paper we will present an alternative approach to calculating the first- and second-order piezoelectric coefficients. This will significantly improve the agreement with experimental data obtained for InAs/GaAs quantum wells compared to the predictions obtained using the parameters proposed by Bester $e t$ $a .^{9}$

\section{PIEZOELECTRIC QUANTUM WELLS}

The piezoelectric effect in III-V semiconductors originates from the lack of inversion symmetry in the zinc blende and wurtzite crystal structures. ${ }^{11}$ Shear strain in such crystals gives rise to a dipole charge distribution. The polarization components are given by $P_{i}=e_{i j k} \varepsilon_{j k}$, with $e_{i j k}$ being the piezoelectric coefficients and $\varepsilon_{j k}$ representing the components of the strain tensor. The only nonzero values of $e_{i j k}$ in zinc blende structures are those associated with the off-diagonal components of the strain tensor $\left(e_{14}, e_{25}, e_{36}\right)$. Since these elements are all equal by symmetry, they can be written in reduced coordinates as a single coefficient usually called $e_{14}$. The expression for the piezoelectric charge originating from the presence of shear strain is then given by

$$
\rho(\mathbf{r})=-\nabla \cdot\left(2 e_{14}(\mathbf{r}) \cdot\left[\varepsilon_{y z}(\mathbf{r}) \mathbf{i}+\varepsilon_{x z}(\mathbf{r}) \mathbf{j}+\varepsilon_{x y}(\mathbf{r}) \mathbf{k}\right]\right) .
$$

The off-diagonal components of the strain tensor $\varepsilon_{y z}, \varepsilon_{x z}$, and $\varepsilon_{x y}$ are nonzero for strain in the [111] direction. Quantum wells (QWs) grown on (111) substrates are particularly interesting nanostructures since the induced piezoelectric field lies in the growth direction which simplifies the modeling of experimental data. Previously deduced values of $e_{14}$ for $\mathrm{In}_{x} \mathrm{Ga}_{1-x}$ As alloys ${ }^{12-14}$ from photocurrent experiments showed that $e_{14}$ cannot be fitted by linear interpolation between the bulk values of GaAs and InAs for $x<0.3$.

By modeling the low-temperature photocurrent spectrum of biased QW structures both Hogg et al. ${ }^{12}$ and SánchezRojas et al. ${ }^{13}$ concluded that for several In compositions of $x$ in the range between 0 and 0.3 , a better fit is achieved by using values of $e_{14}$ reduced to around $70 \%$ of the interpolated values. Chan et al. ${ }^{14}$ reported a slightly higher value at room temperature, corresponding to around $80 \%$ of the interpolated value, caused by the simultaneous effects of piezoelectricity and pyroelectricity, ${ }^{15}$ which was also reported by Cho et al. ${ }^{16}$ The calculations used to deduce the reported values of $e_{14}$ in Refs. 12-16 all assumed ideal structures consisting of quantum wells (QWs) with sharp interfaces, using the In composition $x$ and well width as fitting parameters. 


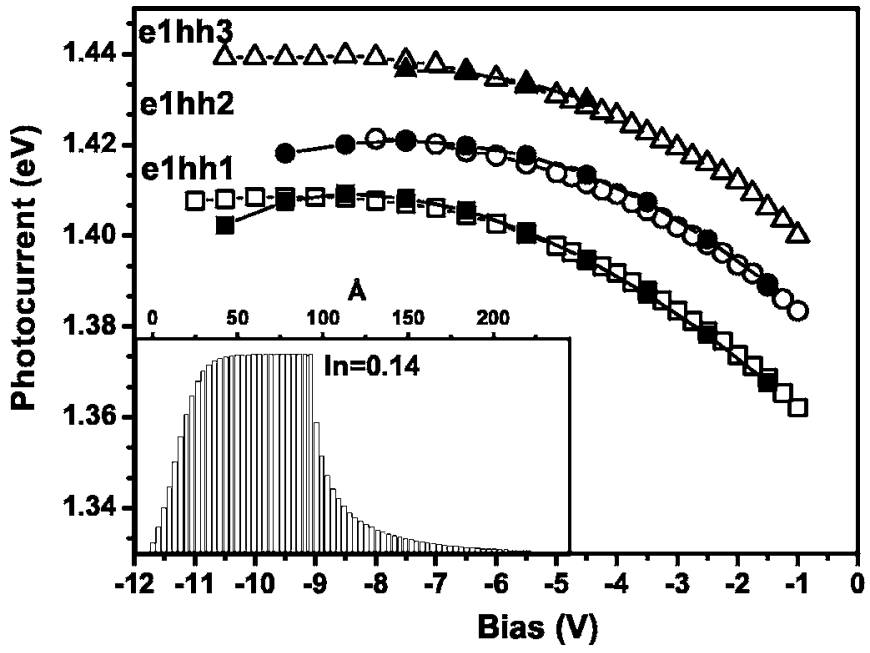

FIG. 1. Experimental excitonic transitions of a (111)B $p-i-n$ GaAs diode containing a 100 - $\AA$-wide, nominal $\operatorname{In}_{0.15} \mathrm{Ga}_{0.85}$ As single quantum well, from a photocurrent experiment ${ }^{19}$ plotted against reverse applied bias (hollow symbols) and the result of the simulation (filled symbols). The best fit was obtained with $x=14 \%$, well width of $103 \AA, e_{14}$ reduced to $84 \%$, and segregation energy $E_{s}$ equal to $0.22 \mathrm{eV}$. In the inset we show the calculated composition profile of the quantum well and the nominal GaAs region above. Notice the segregation profile.

In later work Ballet et al. ${ }^{17}$ pointed out that in similar structures grown by molecular bean epitaxy (MBE) on (001) substrates, In segregation ${ }^{18}$ is known to grade the heterostructure interfaces. Since the same effect can be expected in structures grown on (111) substrates, the authors repeated earlier calculations ${ }^{12}$ including the effects of In segregation. It was found that a better fit can be obtained assuming $e_{14}$ to be $86 \%$ of the interpolated value for $x=0.15$ (corresponding to $-0.124 \mathrm{C} \mathrm{m}^{-2}$ ).

\section{MODELING THE PHOTOCURRENT SPECTRA}

The results presented in this work confirm the prediction of Ballet et al. ${ }^{17}$ Using a similar method we have modeled the experimental ${ }^{19}$ low-temperature $(5 \mathrm{~K})$ photocurrent spectra for biases between $-1 \mathrm{~V}$ and $-11 \mathrm{~V}$ in an MBE-grown (111)B $p-i-n$ GaAs diode containing a nominally $100-\AA \AA$ wide $\operatorname{In}_{0.15} \mathrm{Ga}_{0.85} \mathrm{As}$ single QW. We included the effects of segregation by evaluating the composition of each monolayer (ML) in the QW region using the thermodynamic model of segregation of Dehaese et al. ${ }^{18}$ with values of the segregation energy $E_{s}$ of $0.22 \mathrm{eV} .{ }^{20}$ The resulting characteristic graded compositional profile (see inset in Fig. 1) gives rise to two major effects: First, the confining potential and hence the energy levels are very different compared to those of an ideal interface. Second, the elastic and dielectric properties are also altered upon the grading of the $\mathrm{QW}$ region. We incorporated these effects by a layerwise decomposition of the QW region and the application of continuum-elasticity theory to each of them. To be more precise, we determined the strain for epitaxial layers of $\operatorname{In}_{x} \mathrm{Ga}_{1-x}$ As grown on GaAs (111) substrates as a function of the composition $(x),{ }^{11}$

$$
\begin{aligned}
\varepsilon_{\alpha \beta}= & \left(\delta_{\alpha \beta}-\frac{1}{3}-\frac{1}{3} \frac{2 C_{11}(x)+4 C_{12}(x)-4 C_{44}(x)}{C_{11}(x)+2 C_{12}(x)+4 C_{44}(x)}\right) \\
& \times\left(\frac{a_{\text {InGaAs }}(x)}{a_{\text {GaAs }}}-1\right),
\end{aligned}
$$

in terms of the composition-dependent lattice $a$ and elastic constants $C_{i j}$. To write Eq. (2) in compact form we represented the Cartesian coordinates through the symbols $\alpha$ and $\beta$ and made use of the Kronecker delta $\left(\delta_{\alpha \beta}\right)$. In order to evaluate the piezoelectric field in the QW region one must also consider the absolute value of polarization for each layer given by ${ }^{11}$

$$
P_{\text {piezo }}=2 \sqrt{3} \varepsilon_{i j} e_{14}(x)
$$

and make use of the continuity relation for the displacement vector $\mathbf{D}=\varepsilon_{0} \mathbf{E}+\mathbf{P}$ among adjacent layers. The solution to the resulting system of linear equations is a spatially nonuniform piezoelectric field that can then be used to evaluate the built-in slope of the confining potential in the QW region. Finally, we calculated the exciton binding energies as a function of the external field created by the sample bias. From our calculated photocurrent spectrum we have found that an excellent fit to the first few exciton binding energies (Fig. 1) could be obtained by using $x=0.14$ for the QW composition, using a QW width of $103 \AA$ (this is a 3\% difference compared to the nominal QW width of $100 \AA$ ), and a reduction of $e_{14}$ to $84 \%$ of the interpolated value (at $x=0.14$ ). Here, we assumed a linear scaling for the intermediate compositions between the former composition and $x=0$ (GaAs).

We observed that an accurate fit to the experimental data of even a single sample is only possible for an interpolation of $e_{14}$ that takes the stoichiometry profile that arises from the segregation (see inset in Fig. 1) into account.

The presence of In segregation in MBE-grown (111)B QWs has also been observed directly by Moran et al. ${ }^{20}$ with energy-filtered transmission electron microscopy and was found to be consistent with the thermodynamic model of segregation. ${ }^{18}$ Such direct determination leaves little doubt that for MBE-grown samples, only the values of $e_{14}$ that were deduced by taking the segregation effect into account are likely to be realistic. Samples grown by MOVPE have been studied by Cho et al., ${ }^{16}$ who have measured the temperature dependence of $e_{14}$. They assumed sharp interfaces and discussed both the low-temperature behavior and the pyroelectric contribution at higher temperatures, which increases the estimated value of the piezoelectric coefficient. The values of $e_{14}$ obtained at low temperature are around $70 \%$ of the interpolated values and are similar to the values deduced by Sánchez-Rojas et al. ${ }^{12}$ In the absence of a direct composition measurement this suggests either that the heterojunction interfaces are graded or that some other structural parameter (in Ref. 16 these were determined by highresolution $\mathrm{x}$-ray diffractometry) may be inaccurate.

\section{MODELING OF THE PIEZOELECTRIC COEFFICIENT}

From our fitted data and that of Ballet et al. ${ }^{17}$ we conclude that the low temperature values of $e_{14}$ for $0 \leq x \leq 0.2$ are 
around $85 \%$ of the linearly interpolated values. In this section we discuss the values of $e_{14}$ at higher In concentrations. Unfortunately it is not possible to extend the experiments described in Refs. 12-17, 19, and 20 to In concentrations of $x>0.4$, since the large lattice mismatch degrades the crystal quality. In order to make any attempt to predict the values of $e_{14}$ for $x>0.2$ we first need to understand the observed reduction from the linearly interpolated values. We are therefore going to present a microscopic description of the elastic and charge distributions that are at the origin of the piezoelectric effect in III-V semiconductors.

\section{A. Model for the piezoelectric polarization}

We follow the model proposed by Harrison ${ }^{21}$ that was originally based on the bond orbital approximation. In this model one considers a single tetrahedron (five atoms) that is subject to small shear strain. Under such conditions, the cation and anion sublattices undergo a further displacement relative to each other to minimize the cohesive energy. This relaxation occurs in the direction perpendicular to the shear strain, e.g., along the $\boldsymbol{k}$ direction if the shear strain is in the $\boldsymbol{i} \boldsymbol{j}$ plane, as initially pointed out by Kleinman. ${ }^{22}$ This displacement $\delta \boldsymbol{k}$ is assumed to be linear in the strain and can therefore be characterized by a parameter $\zeta$, which is normally $<1$ and assumed to be constant for a given material. It can be easily shown that $\delta \boldsymbol{k}$ can be written as $a \zeta \varepsilon_{i j} / 4$, with $a$ the lattice constant and $\varepsilon_{i j}$ the shear strain. This displacement directly contributes to the piezoelectric charge, as cations and anions possess an effective charge of the opposite sign. Furthermore, due to the overall displacement of the sublattices, the four nearest neighbors of the atoms at the center of the tetrahedron have a different bond length compared to the unstrained case, which results in an additional dipole moment on each bond. These contributions can be added using simple geometrical arguments and are counteracting as the direct and the dipole contribution have opposite sign. The comparably small strains in most semiconductor heterostructures allow us to approximate the modifications of the bond lengths and the bond angles in linear order of the strain and the resulting expression for the polarization due to the piezoelectric charges (in units of the electron charge) reads

$$
\begin{aligned}
p_{\hat{k}} & =Z_{H}^{*} \delta \hat{k}+\beta \sum_{q=1,4}\left|\vec{r}_{q}\right| \cos \theta_{\vec{r} q} \hat{k} \\
& =Z_{H}^{*} \frac{a \zeta \varepsilon_{i \hat{j}}}{4}+\beta \frac{a \sqrt{3}(1-\zeta) \varepsilon_{i \hat{j}}^{\hat{j}}}{3} \frac{4}{3} \cos \theta_{\vec{r}_{1}} \hat{k},
\end{aligned}
$$

where $Z_{H}^{*}$ is the atomic effective charge associated with each atom in the crystalline structure, and $\beta$ is the transfer parameter. ${ }^{23}$ The latter is more conventionally written in terms of the bond polarity, which is a measure of the electronic dipole associated with a bond, as $\beta=2 \alpha_{p}\left(1-\alpha_{p}^{2}\right)$. With $r_{q}$ we have indicated the four nearest neighbors of the central atom in the tetrahedron under consideration. The quantity $p_{k}$ in Eq. (4) is the total polarization in units of the electron charge. The absolute polarization per unit volume is

$$
\begin{aligned}
P_{\hat{k}} & =\frac{e}{2 \Omega_{0}} \frac{a \zeta \varepsilon_{i \hat{j}}}{4}\left(Z_{H}^{*}-\frac{4}{3} \alpha_{p}\left(1-\alpha_{p}^{2}\right) \frac{(1-\zeta)}{\zeta}\right) \\
& =\frac{e e_{p}^{*} a \zeta}{V} \varepsilon_{i \hat{j}}=e_{14} \varepsilon_{\hat{i} \hat{j}},
\end{aligned}
$$

where $e_{p}^{*}$ is the piezoelectric charge (which depends on $Z_{H}^{*}$, $\alpha_{p}$, and $\left.\zeta\right), V$ is the volume of the unit cell, and $\Omega_{\mathrm{o}}$ is the volume per atom. Within this model $e_{14}$ is constant for a given material and the piezoelectric effect is linear in the strain.

In the following sections we will describe how large strains affect this simple description, but prior to our discussion we would like to clarify the theoretical background of this approach.

In this work we follow the decomposition of polarization effects proposed by Harrison ${ }^{21}$ that can be regarded as a first-order approximation with respect to the atomic dipoles. Higher-order effects arising from the dipoles of distant neighbors are not included. As a result, this approximation introduces some element of fitting in determining the bulk values of $Z_{H}^{*}$, as we shall discuss in Sec. IV C. Furthermore, the quantities $Z_{H}^{*}$ and $\alpha_{p}$ cannot be measured or calculated directly, but are rather tight binding expressions with little physical meaning outside the context of such theory. One can, however, consider these two quantities as fitting parameters, the values of which can be obtained indirectly from methods other than tight binding (e.g., DFT). Therefore the proposed method is legitimate in using Harrison's description of the polarization effects and then incorporating parameters from, e.g., DFT calculations.

\section{B. Internal sublattice displacement}

We used plane-wave pseudopotential density functional theory to evaluate the changes in the internal sublattice displacement, commonly known as the Kleinman parameter, as a function of shear and hydrostatic strain for InAs and GaAs zinc blende structures. The pseudopotentials were derived by employing the Troullier-Martin scheme, ${ }^{24}$ and the electronic exchange-correlation interactions were considered within the local density approximation. ${ }^{25}$ Single-particle orbitals expressed in a plane-wave basis set up to the kinetic energy of $50 \mathrm{Ry}$, and Brillouin zone summations on $4 \times 4 \times 4$ Monkhorst-Pack $k$-point grids ${ }^{26}$ were sufficient to converge the value of the Kleinman parameter $\zeta$ below to a remaining error of about $1 \%$. The theoretical equilibrium lattice constants for GaAs and InAs were 10.48 a.u. and 11.37 a.u., respectively. The strain tensor used had only two independent components: $(1-\varepsilon)$ for the three diagonal components and $\gamma / 2$ for the six off-diagonal components. The dependence on the hydrostatic strain was evaluated by applying a small shear $(\gamma<0.01)$ to the hydrostatically compressed lattice, and then relaxing the anion and cation sublattices. It is easy to show that the resulting sublattice displacement is in this case given by

$$
\delta r=\sqrt{3} \frac{a \zeta \gamma}{4(1+\varepsilon)} .
$$

Figure 2 shows the results for the calculated Kleinman parameter of zinc blende InAs (circles) and GaAs (squares) on 


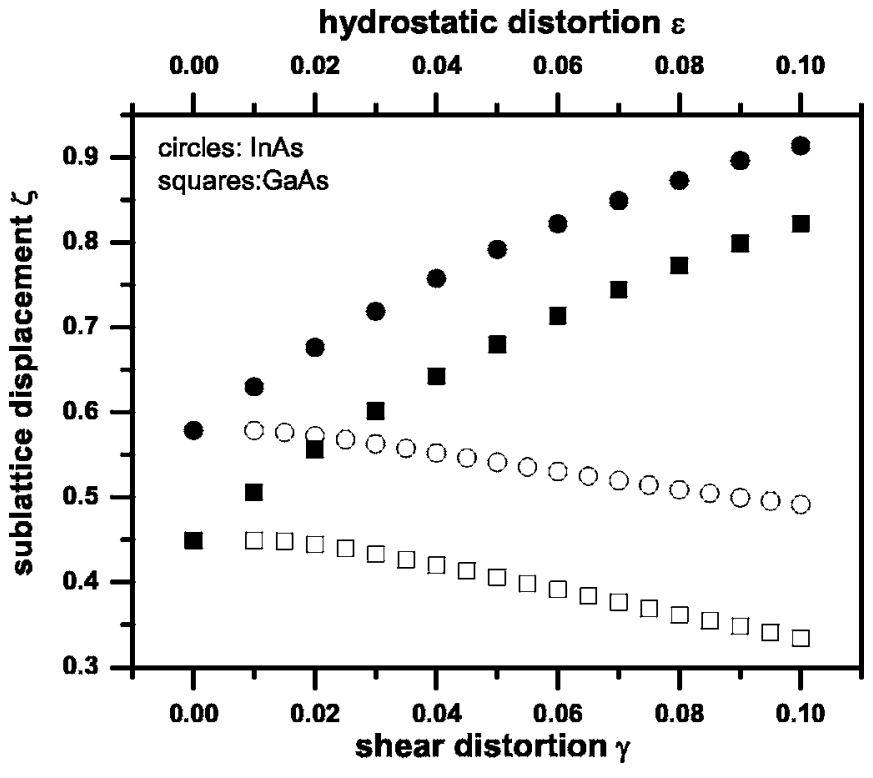

FIG. 2. Dependence of the Kleinman internal displacement parameter $\zeta$ of zinc blende InAs (circles) and GaAs (squares) on shear strain (hollow symbols) and hydrostatic strain (filled symbols). Notice the opposite strain dependence of these two.

shear (hollow symbols) and hydrostatic (filled symbols) strain. Of the small strain values $(0.595$ for InAs and 0.449 for GaAs), only the value for GaAs is slightly lower than the recently reported DFT calculations. ${ }^{27}$ It is obvious that the effect is similar for the two materials and that there is an increase/decrease for larger hydrostatic/shear strain, a trend already reported for hydrostatic strain by Wang and $\mathrm{Ye}^{28}$ while to our knowledge no one has so far reported the shear strain dependence. It is worth noting that according to Eq. (5), increasing $\zeta$ leads to reduced piezoelectric charges while decreasing $\zeta$ results in the opposite effect.

Further DFT calculations ${ }^{29}$ were performed to assess the influence of coupling large hydrostatic and shear strain. We found that in this case the values of $\zeta$ are not very different (the difference is smaller than $0.6 \%$ ) than the values obtained by adding the effects of hydrostatic and shear separately. In any case the difference was fitted and the coefficients are given in Table I.

This calculation clearly shows how some of the assumptions of Harrison's theory ${ }^{21}$ break down in the limit of large strains. In particular, the theory of piezoelectricity ${ }^{21}$ relied upon the assumption that the Kleinman parameter is constant under shear strain. For small values of $\gamma(<0.02)$ this is an acceptable approximation, but larger strain can change this elastic property by as much as $35 \%$ for $\gamma=0.1$ (Fig. 2).

\section{Bond polarity $\alpha_{p}$ and atomic effective charge $Z_{H}^{*}$}

The most recent calculation for $\alpha_{p}$ by Wang and $\mathrm{Ye}^{28}$ employed density functional perturbation theory to evaluate the transverse effective charge and then determined $\alpha_{p}$ from tight binding expressions that link the two quantities. The reported values are 0.49 and 0.423 for InAs and GaAs, respectively, ${ }^{30}$ close to the values reported by Shen, ${ }^{31}$ ob-
TABLE I. Parameters used in our calculations.

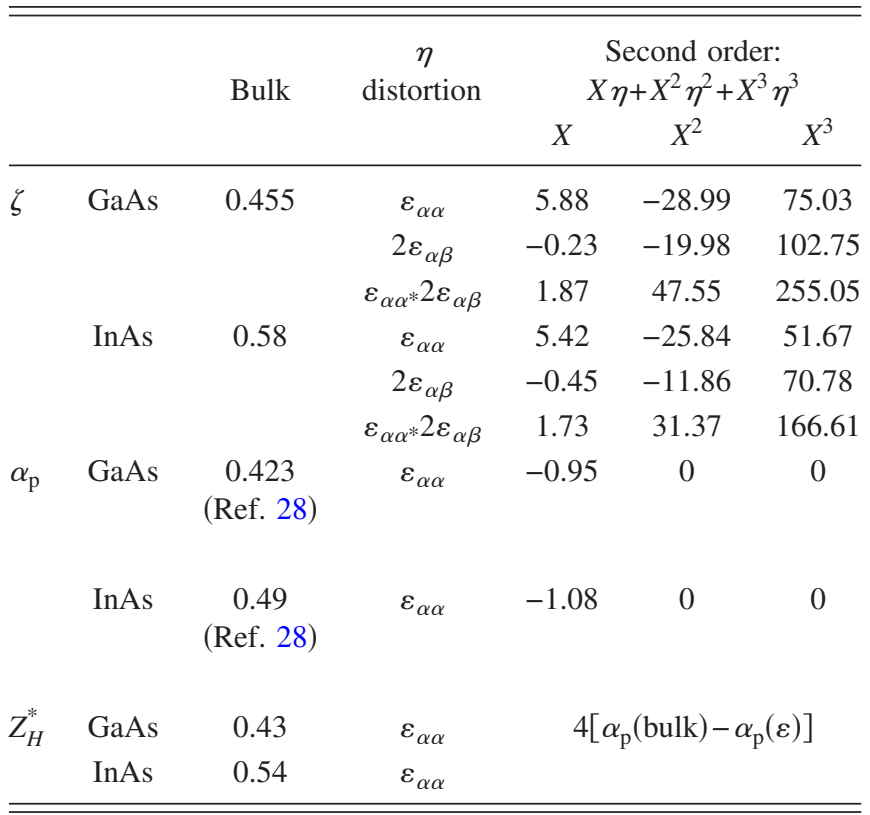

tained with a bond orbital approximation method. Wang and $\mathrm{Ye}^{28}$ also evaluated the effect of compressive hydrostatic strain, which is shown to linearly reduce the polarity with an estimated decay of $-1.08 \varepsilon$ and $-0.95 \varepsilon$ for InAs and GaAs, respectively.

Obtaining accurate values for the atomic effective charge $Z_{H}^{*}$ is not straightforward. Harrison ${ }^{21}$ suggested that $Z_{H}^{*}=Z$ $-4+4 \alpha_{p}$, where $Z$ is the chemical species column number, but data extrapolated from experiment by Falter et al. ${ }^{32}$ suggests that this approach tends to overestimate $Z_{H}^{*}$. Several studies $^{31-33}$ have reported values for the effective charge $Z^{* 23}$ and show close agreement with experimental data. Though in theory $Z^{*}=Z_{H}^{*}+4 \beta / 3$, the latter expression is likely to be a crude approximation ${ }^{34}$ and so far it has proven difficult to obtain widely agreed values of $Z_{H}^{*}$. Given the rather large spread of experimental and theoretical data, we decided to follow a simple approach. We applied Eq. (5) (using the DFT calculated equilibrium lattice constants) and DFT values of the polarity $\alpha_{p}$ and $\zeta$ while using $Z_{H}^{*}$ as a fitting parameter to ensure that Eq. (5) gives the correct bulk InAs and GaAs $e_{14}$ experimental values of -0.045 and $-0.16 \mathrm{C} / \mathrm{m}^{2}$, respectively. Using this procedure we obtained corresponding values of $Z_{H}^{*}$ of 0.54 and 0.43 , which are in reasonable agreement with those (0.612 and 0.323$)$ of a reported minimal basis tight binding calculation. ${ }^{35}$

As pointed out by Wang and $\mathrm{Ye},{ }^{28}$ the effective charges also must depend on the hydrostatic strain, and as it is extremely difficult to obtain such dependence reliably, we used the fact that in first approximation

$$
Z_{H}^{*}=Z-4+4 \alpha_{p}
$$

In fact, though the latter expression has been shown ${ }^{31,32}$ to overestimate $Z_{H}^{*}$, the difference given by $Z_{H}^{*}-Z_{H}^{*}(\varepsilon)$ is a reasonable approximation for the effects of the hydrostatic strain, and hence we can derive the strain dependence of $Z_{H}^{*}$ from the expression for $\alpha_{p}(\varepsilon)$. 


\section{Effects of compositional disorder}

For intermediate compositions between GaAs and InAs in first approximation it is common to use a linear interpolation scheme between bulk values. However, it has been shown by Bouarissa, ${ }^{36}$ who used the empirical pseudopotential method within the virtual crystal approximation, that such a scheme is not correct for quantities such as the polarity, which for around $x=0.5$ is calculated to be $8 \%$ larger than the interpolated value. This effect is linked to compositional disorder in random alloys. By using the information provided in Ref. 4 we derived a bowing parameter and therefore we can write the composition-dependent polarity through

$$
\alpha_{p}=0.423+0.161 x+0.00148 x^{2} .
$$

Again, for $Z_{H}^{*}$ we follow a similar scheme to that used to obtain the strain dependence, that is, using the polarity values from Eq. (8) into Eq. (7).

Elastic properties for $\operatorname{In}_{x} \mathrm{Ga}_{1-x}$ As alloys are known to follow Vegard's law, and hence we do not need to include any bowing parameter for the Kleinman parameter.

\section{E. Evaluation of the piezoelectric coefficient}

In order to evaluate the piezoelectric coefficient in the case of a hydrostatically and shear deformed crystal, we introduce several modifications to Eq. (5). If one starts from Eq. (4), it becomes obvious from Eq. (6) that the direct contribution needs to be divided by $(1-\varepsilon)$, and that, to first order in the strain, the cell volume becomes $V^{\prime} \cong V(1+3 \varepsilon)$. In order to compare with existing experimental data we are required to evaluate the piezoelectric coefficient for intermediate compositions of $\operatorname{In}_{x} \mathrm{Ga}_{1-x}$ As. Therefore, we linearly interpolate the quantities $a$ and $\zeta$ while using the scheme described in the previous section for $\alpha_{p}$ and $Z_{H}^{*}$. The obtained value for the piezoelectric coefficient is the linear term (see Fig. 3) and it is dissimilar to a simple linear interpolation between the bulk values of $e_{14}$. In order to compare with the experimental data we further need to incorporate the strain effects. We have therefore evaluated, using Eq. (2), the strain for a uniform epitaxial layer of $\operatorname{In}_{x} \mathrm{Ga}_{1-x}$ As grown on GaAs (111) substrates as a function of the composition $(x)$.

We have then evaluated the changes in the interpolated microscopic quantities $\zeta, \alpha_{p}$, and $Z_{H}^{*}$ as a result of the applied strain. This was achieved using fitted and then interpolated parameters obtained from the data contained in Fig. 2 and the relationships described in Secs. IV B-IV D. We then used the modified Eq. (5) to evaluate $e_{14}$. A summary of the scheme used for the strain dependence is given in Table I.

\section{DISCUSSION}

The results obtained with our model are shown in Fig. 3, together with the previous data in the literature, the predicted linear coefficients from the model of Bester et al. ${ }^{9}$ and the result of our fit on the photocurrent spectrum. For the latter we also show points corresponding to the proposed linear regression for small values of the composition $x$.

The strain considerably modifies the values of $e_{14}$. Our calculations show that the largest effect is the modified elas-

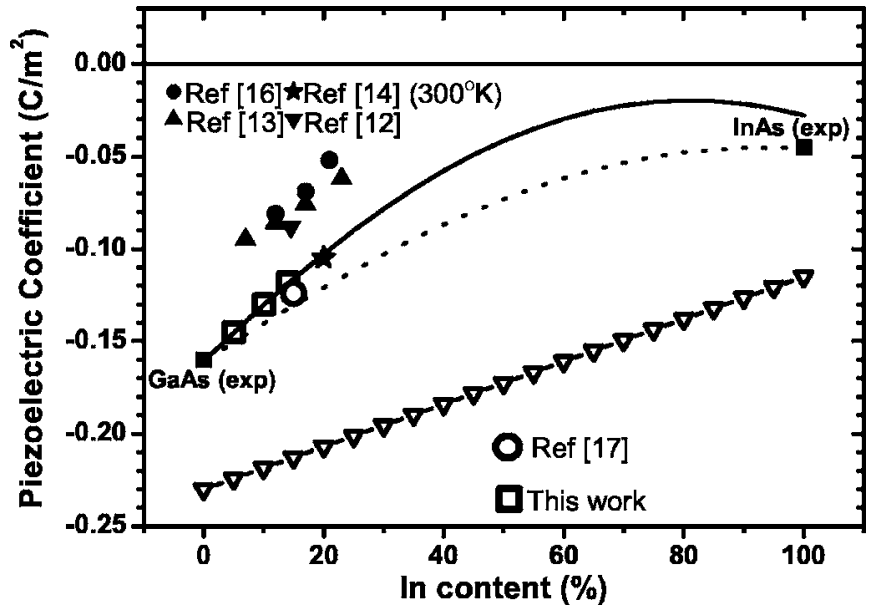

FIG. 3. Piezoelectric coefficient dependence upon In fraction. Data reported in literature is shown (filled symbols), together with the data presented in this work and in Ref. 17 (hollow squares and circle, respectively). The linear term in our model (dotted line) is fitted to the bulk experimental values of GaAs and InAs, while the $a b$ initio calculated linear term of Ref. 9 (open triangles) is substantially larger than the latter. Also, when including the second-order strain effects, our model (line) accurately fits the available experimental data (hollow squares and circle).

tic behavior, and in particular, the change in the Kleinman parameter which is modified by both shear (which reduces it) and hydrostatic (which increases it) strain, with the latter being the dominating effect.

Our model is clearly in close agreement with the values extracted from photocurrent experiments when including the effect of In segregation. Note: The modified piezoelectric coefficient plotted in Fig. 3 is not the value of the piezoelectric coefficient as a function of composition but rather as a function of the strain that a sufficiently thick epilayer of uniform composition $x$ would undergo when grown on a GaAs (111) substrate. Therefore, the value for unstrained InAs bulk shown in Fig. 3 does not overlap with the plotted quantity of strained InAs on GaAs (111).

One obvious difference between our model and that of Bester et $a l .{ }^{9}$ is in the linear term. While the linear term in this work is made to reproduce the experimental values of $e_{14}$ for the binary compounds GaAs and InAs, the linear term of Bester et al..$^{9}$ predicts substantially larger values. The model proposed in this work could, in principle, be made to agree with the linear term of Bester et al., ${ }^{9}$ which would be achieved by simply choosing $Z_{H}^{*}$ as 0.2 for both InAs and GaAs (curiously, these are also the experimental values reported by Falter et al. ${ }^{32}$ ). In this case, however, the predicted values for the strained crystals would not agree with the experimental data.

The predictions from this model cannot readily be compared with the results of the model of Bester et al. ${ }^{9}$ when including the second-order strain effects, as the authors discussed in detail the values of the piezoelectric field rather than those of the piezoelectric coefficient. The experimental values of the piezoelectric coefficient for indium compositions of the QW between $x=0.15$ and $x=0.21$ have been reported in Refs. $12-17$ to be in the range $120-165 \mathrm{kV} / \mathrm{cm}$. 
However, such values could only be obtained indirectly from electro-optical measurements and are largely dependent on the assumptions made regarding the structural parameters of the QWs used in the experiments. As an example, when measuring the oscillator strength as a function of the applied bias, it is often assumed that the maximum is reached when the applied bias compensates the internal piezoelectric field (flatband condition). However, this relies on the assumption that the band gap is constant throughout the well region, while if the stoichiometry profile is nonuniform, then the maximum of the oscillator strength and the flatband condition occur at different values of the applied bias.

The fact that the estimated values of the piezoelectric field are often unreliable was clearly pointed out by Ballet et al.: ${ }^{17}$ in a 10 -nm-wide $\mathrm{QW}$, for $x=0.15$, the piezoelectric field is $220 \mathrm{kV} / \mathrm{cm}$ using the theoretical expression, ${ }^{11} 165 \mathrm{kV} / \mathrm{cm}$ if assuming a $30 \%$ reduction of $e_{14}$ from the interpolated value, and $190 \mathrm{kV} / \mathrm{cm}$ when assuming a $16 \%$ reduction of $e_{14}$ together with diffused interfaces (In segregation). This shows that In segregation is responsible for a large part of the discrepancy between the reported values of $e_{14}$. For the case of diffused interfaces the authors reported the largest value of the field, as the latter is nonuniform through the QW structure due to differences in the composition profile.

In an 11-nm-wide $\mathrm{QW}$, for $x=0.15$, the value of the piezoelectric field predicted by Bester et al. ${ }^{9}$ is about $80 \mathrm{kV} / \mathrm{cm}$. This is smaller than the value reported in Refs. $12-14$ and is $42 \%$ of the value indicated by Ballet et al. ${ }^{17}$ to be the most realistic estimate. Our model instead predicts values of the piezoelectric coefficient that results in a field that matches closely that of Ballet et al. ${ }^{17}$ This is clearly because the methods used to evaluate the field are identical and the values of $e_{14}$ are very similar.

The origin of this discrepancy between our model and that of Bester et al. ${ }^{9}$ is not clear. It is not possible, based on the published data, to compare directly the microscopic effects of our model with those of the self-consistent DFT and the linear response technique. The model of Bester et al. ${ }^{9}$ has the merit of correctly explaining qualitatively the origin of the discrepancies found in the literature. However, the close agreement between our predictions and the available experimental data indicates that our model is also quantitatively correct due to the accurate reproduction of the second-order effects in the strain to the piezoelectric polarization.

\section{CONCLUSIONS}

In conclusion, we have presented a general model to explain the strain dependence of the piezoelectric coefficients in semiconductor alloys. We have shown how the piezoelectric coefficient can be quantitatively determined using DFT calculated values for structural and electric charge parameters. The model was applied to $\operatorname{In}_{x} \mathrm{Ga}_{1-x}$ As alloys and was shown to produce predictions that are in close agreement with experimental data for quantum well structures on (111) substrates.

\section{ACKNOWLEDGMENTS}

The authors would like to thank Graham Rees, Martin Moran, and John Roberts from the University of Sheffield for discussions about the piezoelectric quantum well structures; Andrei Schliwa and Robert Seguin from the TU-Berlin for supplying the compiled data of the previously reported values of $e_{14}$; and Alex Zunger and Gabriel Bester (NRELColorado) for very useful discussions on piezoelectricity in strained semiconductors. Thanks also to Luke Wilson from the University of Sheffield for allowing us to reproduce the data from his Ph.D. thesis. This work was supported by the Royal Academy of Engineering UK, the UK Engineering and Physical Sciences Research Council (EPSRC), the SFB296 of the Deutsche Forschungsgemeinschaft, and the European Network of Excellence SANDiE.
*Corresponding author. Email address: M.Migliorato@physics.org

${ }^{1}$ W. G. Cady, Piezolectricity (McGraw-Hill, New York, 1946).

${ }^{2}$ R. M. Martin, Phys. Rev. B 5, 1607 (1972).

${ }^{3}$ M. A. Migliorato, D. Powell, S. L. Liew, A. G. Cullis, M. Fearn, J. H. Jefferson, P. Navaretti, M. J. Steer, and M. Hopkinson, J. Appl. Phys. 96, 5169 (2004);

${ }^{4}$ M. A. Migliorato, D. Powel, E. A. Zibik, L. R. Wilson, M. Fearn, J. H. Jefferson, M. J. Steer, M. Hopkinson, and A. G. Cullis, Physica E (Amsterdam) 26, 436 (2005);

${ }^{5}$ G. Bester and A. Zunger, Phys. Rev. B 71, 045318 (2005).

${ }^{6}$ M. Grundmann, O. Stier, and D. Bimberg, Phys. Rev. B 52, 11969 (1995).

${ }^{7}$ J. H. Davies, J. Appl. Phys. 84, 1358 (1998).

${ }^{8}$ O. Stier, M. Grundmann, and D. Bimberg, Phys. Rev. B 59, 5688 (1999).

${ }^{9}$ G. Bester, X. Wu, D. Vanderbilt, and A. Zunger, Phys. Rev. Lett. 96, 187602 (2006).

${ }^{10} \mathrm{~S}$. Adachi, Physical Properties of III-V Semiconductor Compounds (Wiley, New York, 1992).
${ }^{11}$ E.g., see J. Singh, Physics of Semiconductors and their Heterostructures (McGraw-Hill, New York, 1993).

${ }^{12}$ R. A. Hogg, T. A. Fisher, A. R. K. Wilcox, D. M. Whittaker, M. S. Skolnick, D. J. Mowbray, J. P. R. David, A. S. Pabla, G. J. Rees, R. Grey, J. Woodhead, J. L. Sánchez-Rojas, G. Hill, M. A. Pate, and P. N. Robson, Phys. Rev. B 48, 8491 (1993).

${ }^{13}$ J. L. Sánchez-Rojas, A. Sacedón, F. Gonzáles-Sanz, E. Calleja, and E. Muñoz, Appl. Phys. Lett. 65, 2042 (1994).

${ }^{14}$ C. H. Chan, H. H. Lin, Y. F. Chen, and G. J. Jan, Appl. Phys. Lett. 72, 1208 (1998).

${ }^{15}$ T. B. Bahder, R. L. Tober, and J. D. Bruno, Superlattices Microstruct. 14, 149 (1993).

${ }^{16}$ S. Cho, J. Kim, A. Sanz-Hervás, A. Majerfeld, G. Patriarche, and B. W. Kim, Phys. Status Solidi A 195, 260 (2003); S. Cho, A. Majerfeld, A. Sanz-Hervás, J. J. Sánchez, J. L. Sánchez-Rojas, and I. Izpura, J. Appl. Phys. 90, 915 (2001); S. Cho, A. SanzHervás, J. Kim, A. Majerfeld, and B. W. Kim, J. Appl. Phys. 96, 1909 (2004).

${ }^{17}$ P. Ballet, P. Disseix, J. Leymarie, A. Vasson, A.-M. Vasson, and 
R. Grey, Phys. Rev. B 59, R5308 (1999).

${ }^{18}$ O. Dehaese, X. Wallart, and F. Mollot, Appl. Phys. Lett. 66, 52 (1995).

${ }^{19}$ L. R. Wilson, Ph.D. thesis, University of Sheffield, 1997.

${ }^{20}$ M. Moran, H. Meidia, T. Fleischmann, D. J. Norris, G. J. Rees, A. G. Cullis, and M. Hopkinson, J. Phys. D 34, 1943 (2001).

${ }^{21}$ W. A. Harrison, Electronic Structure and Properties of Solids (Dover Publications, Inc., New York, 1989).

${ }^{22}$ L. Kleinman, Phys. Rev. 128, 2614 (1962).

${ }^{23}$ For $Z^{*}$ the subscript $H$ indicates that for this quantity we follow Harrison's definition (Ref. 15), not to be confused with the commonly used quantity $Z^{*}$, the effective charge, which is instead equivalent to Harrison's $e_{T}^{*}$.

${ }^{24}$ N. Troullier and J. L. Martins, Phys. Rev. B 43, 1993 (1991).

${ }^{25}$ J. P. Perdew and A. Zunger, Phys. Rev. B 23, 5048 (1981).
${ }^{26}$ H. J. Monkhorst and J. D. Pack, Phys. Rev. B 13, 5189 (1976).

${ }^{27}$ S. Q. Wang and H. Q. Ye, Phys. Status Solidi B 240, 45 (2003).

${ }^{28}$ S. Q. Wang and H. Q. Ye, J. Phys. Condens. Matter 17, 4475 (2005).

${ }^{29}$ S. Boeck, A. Alsharif, A. Dick, L. Ismer, A. Qteish, and J. Neugebauer, http://www.sfhingx.de.

${ }^{30}$ The reported values were not calculated directly; the authors calculated the $e_{T}^{*}$ charge, from which $\alpha_{\mathrm{p}}$ can be easily deduced.

${ }^{31}$ San-Guo Shen, J. Phys. Condens. Matter 6, 8733 (1994).

${ }^{32}$ C. Falter, W. Ludwig, M. Selmke, and W. Zieran, Phys. Lett. 105, 139 (1984).

${ }^{33}$ M. Di Ventre and P. Fernández, Phys. Rev. B 56, R12698 (1997).

${ }^{34}$ P. Vogl, J. Phys. C 11, 251 (1978).

${ }^{35}$ M. Kitamura and W. Harrison, Phys. Rev. B 44, 7941 (1991).

${ }^{36}$ N. Bouarissa, Phys. Lett. A 245, 285 (1998). 